
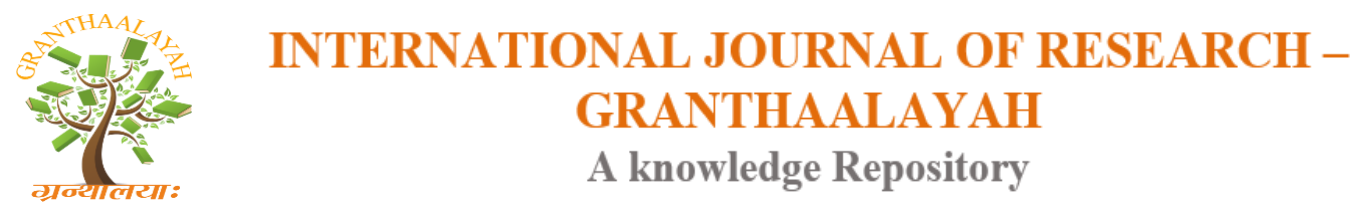

Science

\title{
EVALUATION OF SOME MICROSCOPIC TECHNIQUES FOR DETECTING BILHARZIA AND INTESTINAL PARASITES
}

\author{
Azzam Afifi ${ }^{1}$, Abdel-Aziz A. Ahmed ${ }^{2}$, Yassir Sulieman ${ }^{* 3}$, Mohamed A. Zakaria ${ }^{4}$,
} Theerakamol Pengsakul ${ }^{5}$

${ }^{1}$ Department of Zoology, Faculty of Science and Technology, Omdurman Islamic University, SUDAN

${ }^{2}$ Department of Zoology, Faculty of Science, University of Khartoum, SUDAN

${ }^{* 3}$ Department of Zoology, Faculty of Science and Technology, University of Shendi, SUDAN

${ }^{4}$ Department of Biology, Faculty of Education, University of Nyala, SUDAN

${ }^{5}$ Faculty of Medical Technology, Prince of Songkla University, Hat Yai, Songkhla 90110, THAILAND

DOI: https://doi.org/10.29121/granthaalayah.v4.i7.2016.2611

\section{ABSTRACT}

The objective of the present study was to evaluate the efficacy of five different diagnostic techniques (haematouria, macro-haematouria, syringe filtration, vacuum flask filtration and sedimentation) in the detection of urinary schistosomiasis. Likewise, five techniques (direct smear, cellophane thick smear, ether-formalin, modified Kato-template and modified Katosyringe) techniques used in the detection of intestinal schistosomiasis and other intestinal parasites were evaluated using fecal samples. The results showed that the most efficient diagnostic technique for fecal samples is the modified Kato-Katz technique, while the etherformalin method was the most sensitive tool for the detection of Schistosoma mansoni, Enterobius sp. and Ascaris sp. rates of infection and worm burden. The modified Kato techniques (template and syringe) failed to detect the intestinal protozoa, Giardia lamblia and Entamoeba histolytica, while the syringe filtration technique was the most powerful urine test.

Keywords:

Microscopic; Diagnosis; Evaluation; Schistosomes; Intestinal parasites.

Cite This Article: Azzam Afifi, Abdel-Aziz A. Ahmed, Yassir Sulieman, Mohamed A. Zakaria, and Theerakamol Pengsakul, "EVALUATION OF SOME MICROSCOPIC TECHNIQUES FOR DETECTING BILHARZIA AND INTESTINAL PARASITES" International Journal of Research - Granthaalayah, Vol. 4, No. 7 (2016): 185-195.

\section{INTRODUCTION}

There are many different microscopic diagnostic techniques for detecting schistosomes and intestinal parasites. These techniques vary in their sensitivity in the diagnostic screening of 
developmental stages of such parasites as well as their intensity. Moreover, many excellent diagnostic methods have been developed and tested over the years (Sturrock, 2001). For example, to determine possible economic diagnostic strategies for future intervention programs, Ansong et al., (2011) evaluated urine analysis for detecting the symptoms of urinary schistosomiasis and showed that haematouria and protienuria have a low sensitivity and low specificity compared to egg counts from filtration and microscopy. Schistosomiasis can be diagnosed through the detection of parasite eggs in stool or urine specimens of the final host (Taylor et al., 1988; Colley et al., 2014). Moreover, antibodies and/or antigens detected in blood or urine samples are also indicators of infection (De Jonge et al., 1988, Corstjens et al., 2015). For urogenital schistosomiasis, filtration techniques using nylon, paper or polycarbonate filters are the standard diagnostic technique (Dazo and Biles, 1974; Magnussen et al,, 2001; Fatiregun et al., 2009). Humans with urinary schistosomiasis almost always have microscopic levels of blood in their urine and this can be detected by chemical reagent strips (van Dam et al., 2004). The eggs of intestinal schistosomes can be detected in fecal specimens through a technique using methylene bluestained cellophane soaked in glycerine or glass slides, known as the Kato-Katz technique (WHO, 2016).

The objective of the present study was to evaluate the efficacy of: (1) Haematouria, macrohaematouria, syringe filtration, vacuum flask filtration and the sedimentation techniques in the detection of Schistosoma haematobium; (2) The direct smear, Kato-Katz (Cellophane thick smear), ether-formalin and the modified Kato techniques (template and syringe) in the detection of $S$. mansoni and other intestinal parasites.

\section{MATERIALS AND METHODS}

\subsection{SAMPLE COLLECTION}

Samples were collected from children from El-Rimeila village (urine and feces, $\mathrm{n}=120$ each) and El-Kalakla village (urine and feces, $n=128$ each), Khartoum State, Sudan. The samples were transferred to the Schistosomiasis Research Laboratory, University of Khartoum for further processing.

\subsection{TECHNIQUES USED FOR URINE SAMPLES}

\subsubsection{HAEMATOURIA}

The rate of $S$. haematobium and $S$. mansoni infection was calculated according to the answers of the children investigated to a simple pre-prepared questionnaire.

\subsubsection{MACRO-HAEMATOURIA}

This technique depends on the direct observation of the urine's color to judge the presence of blood in the urine due to the expulsion of eggs from the urinary tract.

\subsubsection{SEDIMENTATION}

For each child investigated, 10-milliliters of urine was placed into a centrifugation tube and centrifuged at $2000 \mathrm{rpm}$ for 3 minutes. Thereafter the supernatant was discarded and the deposit from each tube was placed as drops on three prepared slides and covered with cover-slips, then 
examined using a binocular microscope. The eggs seen on each of the three slides prepared from a tube were counted and recorded as egg count $/ 10 \mathrm{ml}$ of urine.

\subsubsection{SYRINGE FILTRATION}

Blunt-ended forceps were utilized to place a polycarbonate filter paper on the filter-support of a filter-holder. The filter holder was re-assembled and attached to the end of a $10 \mathrm{ml}$ syringe, from which the plunger was removed. The syringe was then filled to the $10 \mathrm{ml}$ mark with a well-mixed urine sample and the plunger replaced. By holding the syringe over a beaker, the urine was slowly passed through the filter. Then, the filter holder was removed and unscrewed. Again, using blunt-ended forceps, the filter was carefully removed and transferred with the face upwards to a clean glass-slide. A single drop of normal saline was added, and the mixture was then covered with a cover-slip. Three slides from each urine sample were prepared by repeating the same procedure. Using a binocular microscope, the entire filter was examined systematically for the presence of $S$. haematobium eggs. The number of the eggs counted per $10 \mathrm{ml}$ of urine was recorded and the average of the three slides was calculated.

\subsubsection{VACUUM FLASK FILTRATION}

A membranous filter-paper was located in the appropriate place in the filtration apparatus and a vacuum pump was used to evacuate the air from the vacuum filtration flask. The urine sample was poured through the membranous filter-paper and the vacuum pump was switched on, when filtration had finished. Using metal forceps, the filter-paper was removed from the holder and placed on a glass plate with the filtered eggs on the filter-paper, facing away from the glass plate. Ninhydrine saturated solution was gradually poured drop-wise to cover the whole filter paper and allowed to dry gradually under fluorescent light for three hours. The processed filter paper was then completely soaked with normal saline before being located on a glass slide and examined under a microscope, to visualize the darkened bilharzia eggs.

\subsection{TECHNIQUES USED FOR FECAL SAMPLES}

\subsubsection{DIRECT SMEAR}

About 25-milligrams of feces were taken using a wooden stick and placed on a clean slide, to which a drop of normal saline was added. The mixture was covered with a cover-slip and pressed until it spread evenly. This procedure was performed in triplicate for each feces sample.

\subsubsection{KATO-KATZ (CELLOPHANE THICK SMEAR)}

A malachite green solution was prepared by adding $1.0 \mathrm{ml}$ of $3 \%$ malachite green solution to 100 $\mathrm{ml}$ of distilled water and $100 \mathrm{ml}$ of glycerin. Thereafter, a 3 x $3 \mathrm{~cm}$ cellophane sheet was placed in a Petri-dish and covered with the prepared solution and left for 24 hours. One gram of feces was taken and pressed through a sieve with a mesh of $150 \mu \mathrm{m}$ pore size. The sieved feces were calibrated via a Pyrpex template holding exactly 40 milligrams of feces. The sieved calibrated feces were then pressed out on a clean glass-slide. This procedure was performed in triplicate. Each prepared slide was then covered with a transparent cellophane sheet and pressed with a finger until the fecal matter spread to cover an area of 20-25 $\mathrm{mm}$ in diameter. The prepared slides were then, examined after two hours using a binocular microscope. The average of the eggcounts on the slides was taken and multiplied by 25 to calculate the eggs per gram of feces (epg).

\subsubsection{MODIFIED KATO (SYRINGE)}


One gram of feces was taken and pressed through a sieve with a mesh of $150 \mu \mathrm{m}$ pore size. The sieved feces were calibrated via a small disposable syringe estimated to hold only 25 milligrams of feces. Thereafter, the sieved sample was pressed out on a clean glass-slide. The procedure was performed in triplicate. Each slide was then covered with another clear slide to form a "sandwich" and gentle pressure was applied with a finger until the fecal matter spread to cover an area of 20-25 $\mathrm{mm}$ in diameter, then immediately examined using a binocular microscope. The average of the egg-counts on the slides was taken and multiplied by 40 to estimate the epg.

\subsubsection{MODIFIED KATO (TEMPLATE)}

The same procedure as described above for the syringe technique. However, the sieved feces were calibrated using a standard template, containing 40 milligrams of feces. The average of the egg-counts on three slides was multiplied by 25 to calculate the epg.

\subsubsection{ETHER-FORMALIN}

One gram of feces was placed in a mortar dish, and $10 \mathrm{ml}$ of formalin (10\%) was added then mixed with a pestle. The mixture was sieved using surgical gauze in a glass funnel and thereafter transferred into a test tube to which three milliliters of ether was added. The test tube was thoroughly shaken, then centrifuged at $2000 \mathrm{rpm}$ for three minutes. The supernatant was discarded and the deposit of each tube was placed on a slide, and one or two drops of Lugol's solution were added. The slides were covered with cover-slips and examined under a binocular microscope. The average of egg-counts on three slides of each feces sample was recorded.

\subsection{DATA ANALYSIS}

Data analysis was carried out using the statistical software, STATISTIX (version 4, USA). A Chi-square test was used to determine the level of significance of the differences and the Scheffe test was used to assess heterogeneity or homogeneity levels. Values were considered significant when $P<0.05$.

\section{RESULTS}

\subsection{BILHARZIA AND INTESTINAL PARASITES AMONG EL-KALAKLA CHILDREN}

Among the different techniques assessed, the ether-formalin method was found to be the most sensitive in detecting the intestinal protozoa G. lamblia and E. hystolitica, while the modified Kato techniques using both, the template and syringe methods failed to detect either of those protozoa (Fig. 1). Excluding dwarf worms, the sensitivity of the diagnostic techniques evaluated varied significantly at $P<0.05$, in the detection of the reported intestinal parasites including bilharzia (Fig. 1). 


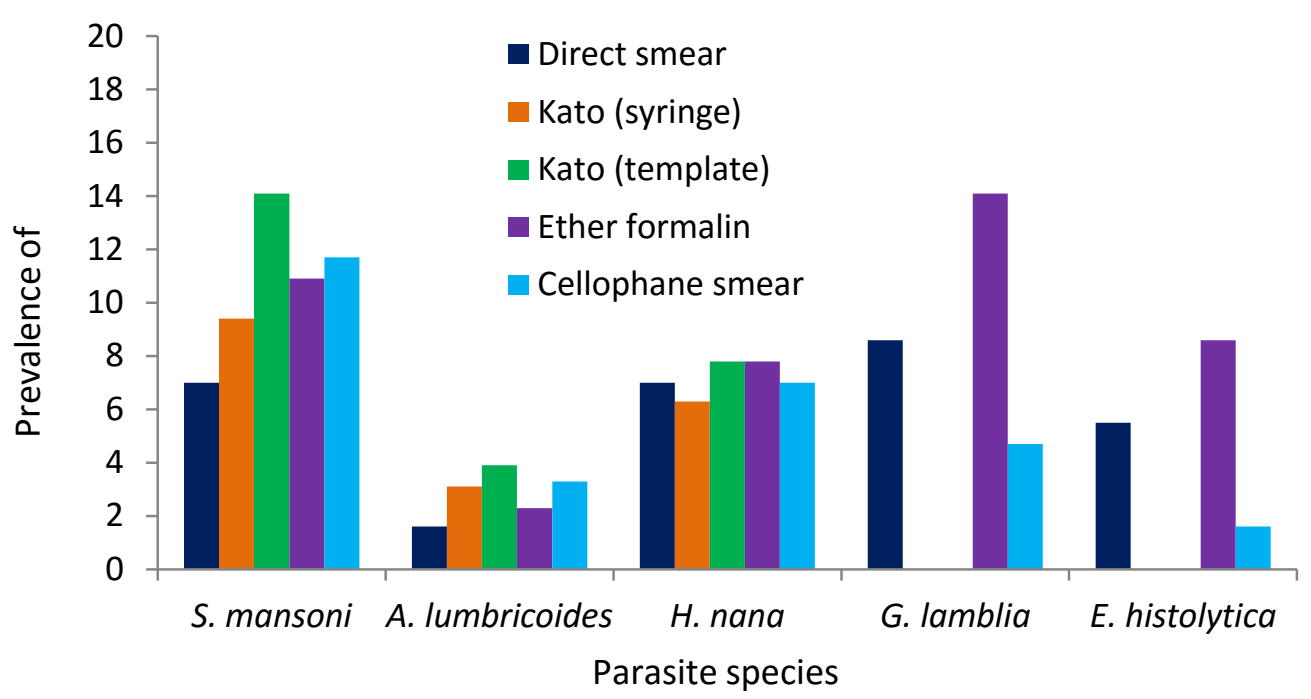

Figure 1: Efficacy of the fecal diagnostic techniques tested in determining intestinal parasites and bilharzia among El-Kalakla children, Khartoum State, Sudan

\subsection{INTENSITY OF BILHARZIA AND INTESTINAL HELMINTHS AMONG EL- KALAKLA CHILDREN}

The most sensitive diagnostic techniques in counting the excreted egg of S. mansoni, A. lumbricoides and Hymenolepis nana were the modified Kato techniques using both the template and syringe methods, while, the direct smear technique was judged to be the poorest tool in counting the excreted eggs of these intestinal worms, (Table 1). The analysis underlined the fact that the diagnostic techniques evaluated varied significantly in their sensitivity for detecting excreted eggs, at $P<0.05$.

Table 1: Efficacy of the screening techniques tested in the detection of the intensity of intestinal helminths and bilharzia among El-Kalakla children, Khartoum State, Sudan

\begin{tabular}{lccc}
\hline \multirow{2}{*}{ Diagnostic technique } & \multicolumn{3}{c}{ Mean number of parasite species eggs excreted \pm SD } \\
\cline { 2 - 4 } & S. mansoni & A. lumbricoides & H. nana \\
\hline Direct smear & $3.4 \pm 2.8$ & $2.6 \pm 1.4$ & $33.5 \pm 2.3$ \\
Syringe modified Kato & $155.4 \pm 3.3$ & $121.2 \pm 1.5$ & $515.4 \pm 2.6$ \\
Template modified Kato & $145.5 \pm 2.9$ & $126.8 \pm 1.7$ & $549.2 \pm 1.5$ \\
Ether-formalin & $56.7 \pm 2.8$ & $57.1 \pm 2.8$ & $317.7 \pm 2.7$ \\
Kato-Katz & $124.8 \pm 2.9$ & $95.6 \pm 2.2$ & $592.7 \pm 1.9$ \\
\hline
\end{tabular}

\subsection{SENSITIVITY OF URINE SCREENING TECHNIQUES AMONG EL-RIMAILA CHILDREN}

The vacuum and syringe filtration techniques were judged to be more sensitive tools, compared to centrifugation technique in counting the number of the released eggs of S. haematobium, and the differences were significant at $P<0.05$. The urine screening techniques in descending order 
of their efficacy in determining the rate of infection were: syringe filtration, centrifugation, vacuum filtration, haematuria and macro-haematuria (Fig. 2).

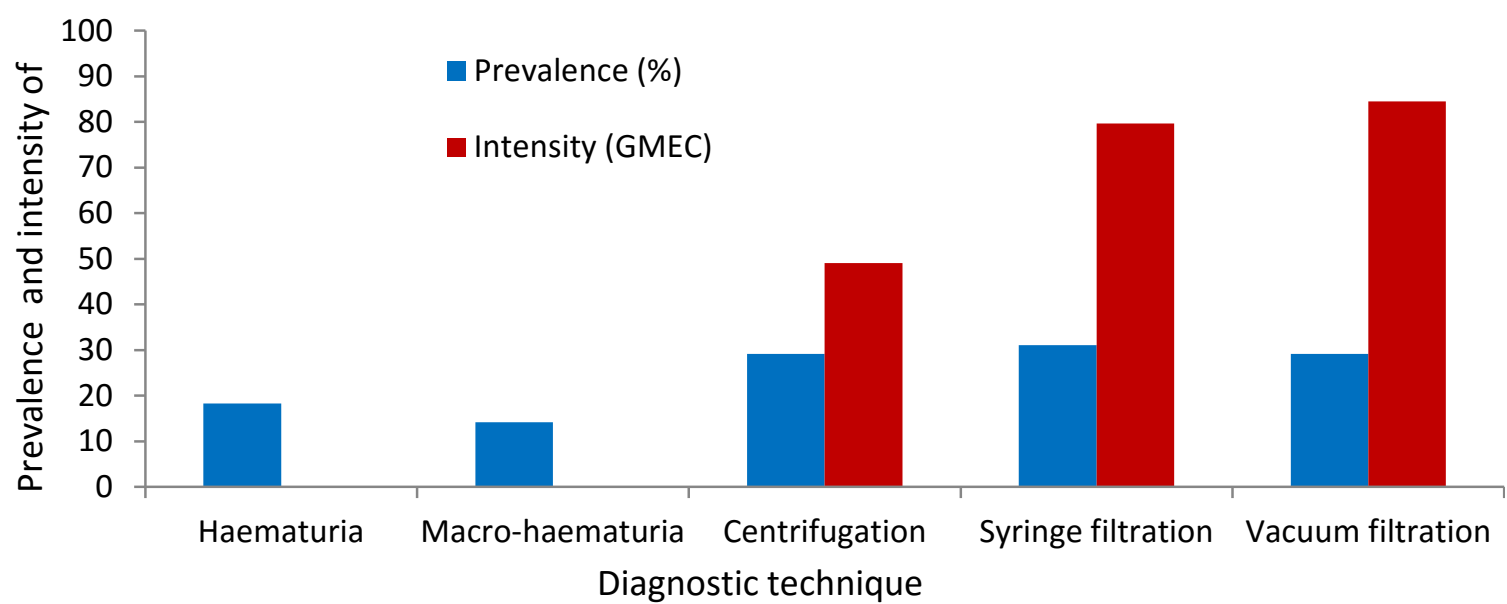

Figure 2: Efficacy of the diagnostic techniques tested in evaluating the rate and intensity of S. haematobium infection among El-Rimaila children, Khartoum State

\subsection{PREVALENCE OF BILHARZIA AND INTESTINAL PARASITES IN EL-RIMAILA}

The most efficient technique in detecting $S$. mansoni ova among children of El-Rimaila was the template modified Kato method, followed by the syringe modified Kato method. On the other hand, the most sensitive diagnostic technique in evaluating the A. lumbricoides infection rate was the template modified Kato method, while the poorest was the direct smear technique. Similarly to the findings in respect of the children at El-Kalakla, the five fecal diagnostic techniques were found to be almost equally effective in detecting $H$. nana eggs. Concerning the detecting of the eggs of the protozoa, Giardia lamblia and Entamoeba histolytica, the ether-formalin, direct smear and Kato-Katz technique were found to have almost equal sensitivity, while the modified Kato techniques, both template and syringe were found not to be able to detect these parasite eggs (Fig. 3). The statistical analysis verifies that the assessed screening techniques varied significantly at $P<0.05$.

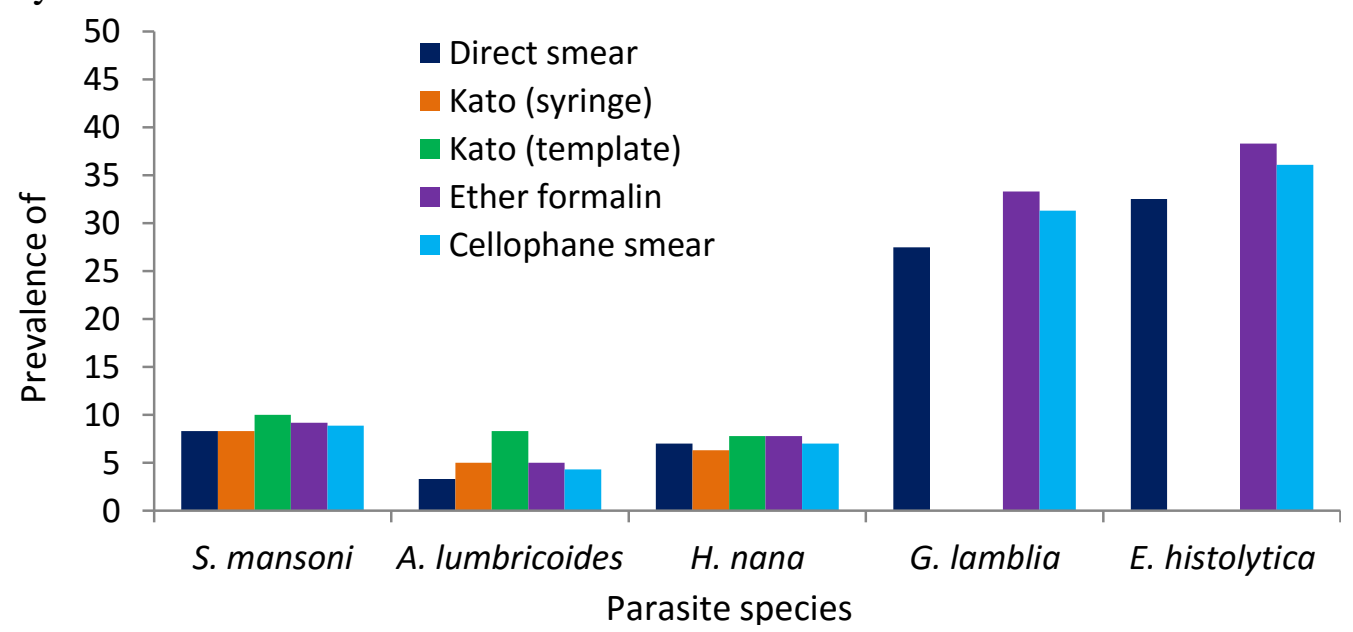

Figure 3: Efficacy of the diagnostic techniques tested in evaluating the rate of bilharzia and intestinal parasite among El-Rimaila children, Khartoum State, Sudan 


\subsection{INTENSITY OF BILHARZIA AND INTESTINAL HELMINTHS AMONG EL- RIMAILA CHILDREN}

Similarly to the children from the El Kalakla area, the most sensitive diagnostic techniques in counting the excreted egg of $S$. mansoni, A. lumbricoides and H. nana were the modified Kato techniques and the Kato-Katz method, while, the direct smear technique was judged to be the poorest tool in counting the excreted egg of these helminths (Table 2). A Scheffe test confirmed that the evaluated diagnostic techniques were significantly heterogeneous in their sensitivity for the detection of the excreted eggs at $P<0.05$.

Table 2: Efficacy of the screening techniques tested in determining the intensity of intestinal helminthes and bilharzia among El-Rimaila children, Khartoum State, Sudan

\begin{tabular}{llll}
\hline \multirow{2}{*}{ Diagnostic technique } & \multicolumn{3}{l}{ Mean number of parasite species eggs excreted \pm SD } \\
\cline { 2 - 4 } & S. mansoni & A. lumbricoides & H. nana \\
\hline Direct smear & $1.9 \pm 1.5$ & $4.6 \pm 1.6$ & $7.8 \pm 2.1$ \\
Syringe modified Kato & $50.4 \pm 1.4$ & $217.6 \pm 1.8$ & $262.8 \pm 2.3$ \\
Template modified Kato & $65.1 \pm 1.6$ & $227.4 \pm 2.1$ & $296.4 \pm 2.6$ \\
Ether-formalin & $13.2 \pm 1.5$ & $121.8 \pm 2.8$ & $229.5 \pm 4.3$ \\
Kato-Katz & $54.6 \pm 1.5$ & $221.5 \pm 2.2$ & $271.3 \pm 2.7$ \\
\hline
\end{tabular}

\subsection{TIME CONSUMED IN CONDUCTING URINE SCREENING TECHNIQUES}

As expected, the haematuria (questionnaire) and the macro-haematuria (glance estimation) techniques took the least time involving as they did, only the detection of the presence of blood in the urine. The syringe filtration and the centrifugation techniques took not more than eight minutes each to conduct, while the vacuum filtration technique took a relative long time to perform (Fig. 4). Deep analysis, using a Scheffe test, established that the diagnostic techniques evaluated varied significantly in the time needed to detect the eggs of $S$. haematobium, at $P<$ 0.05 .

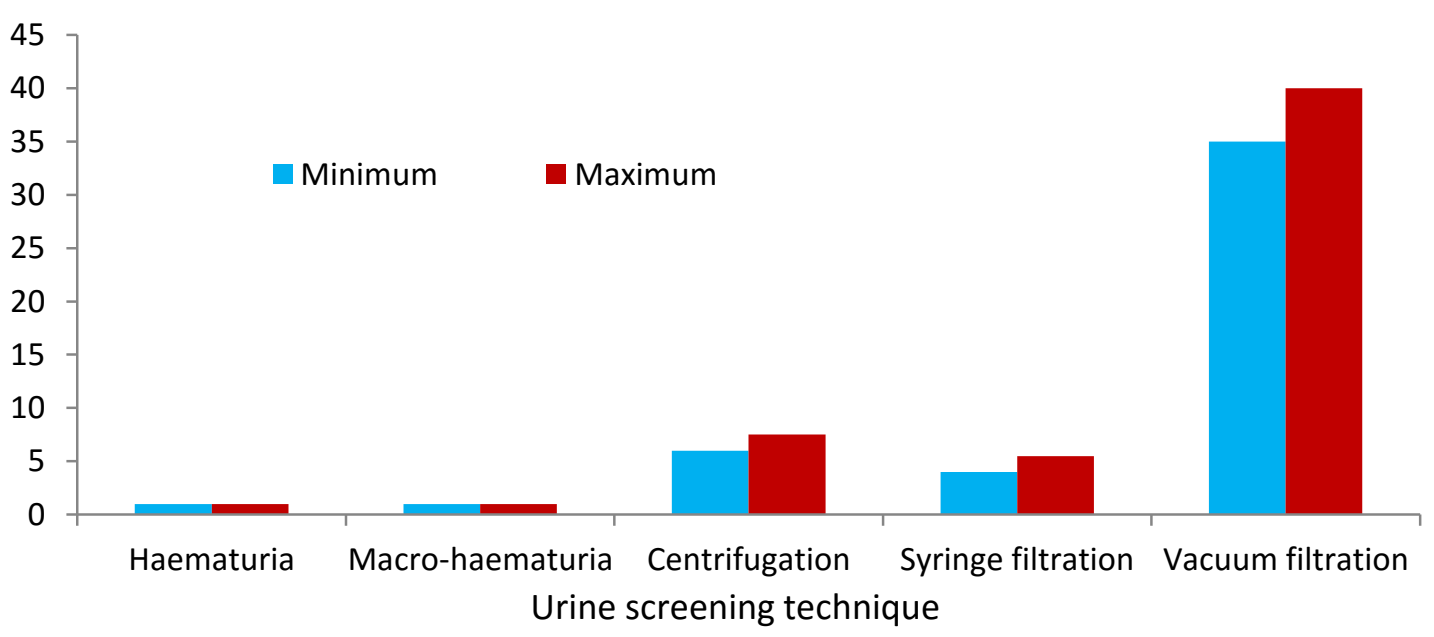

Figure 4: Time taken in detecting S. haematobium using different diagnostic techniques 


\subsection{TIME CONSUMED IN FECES SCREENING TECHNIQUES}

The time taken for the various feces screening techniques fell into three groups. The first included only the direct smear technique which took not more than five minutes. The second consisted of the two modified Kato techniques, template and syringe which took not more than eleven minutes. The third included the ether-formalin and the Kato-Katz techniques which took not more than 15 minutes (Fig. 5). A Scheffe test confirmed that the time interval needed for performing the screening techniques assessed was significantly different at, $P<0.05$.

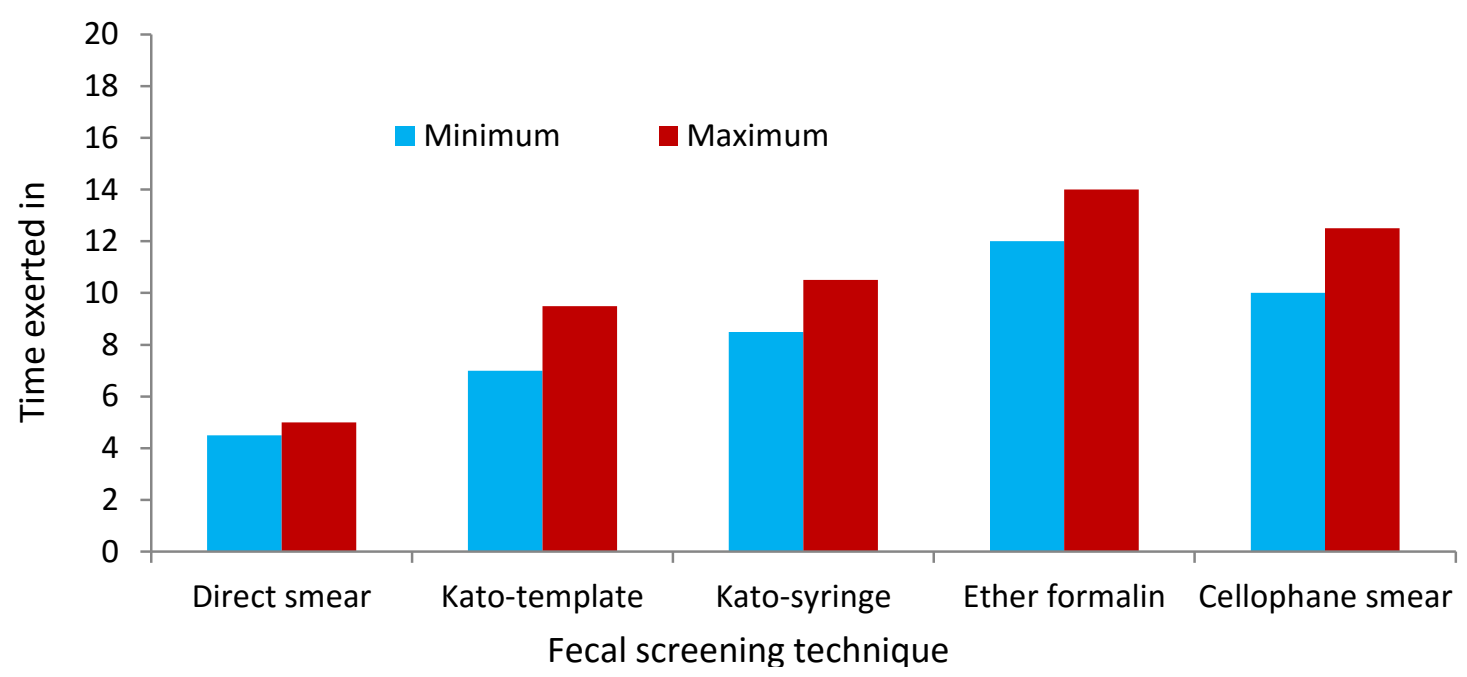

Figure 5: Time exerted in the screening of intestinal parasites using different diagnostic

\section{DISCUSSION} techniques

There are many qualitative and quantitative diagnostic methods available for detecting parasites, but qualitative methods for detecting ova are generally used in clinical practice. All the methods tested in this study for examining urine and stool for helminth eggs have advantages and disadvantages. The time needed for a confident detection of a parasitic infection is a determining factor in the identification of the most efficient microscopic diagnostic techniques.

The macro-haematuria diagnostic technique basically depends on direct observation of the urine's color to judge on the presence of blood in the urine. The presence of blood in the urine is due to the expulsion of eggs from the venules of the urinary bladder. The haematouria and the macro-technique require no more than a glance to detect blood in the urine as an indicator of $S$. haematobium infection (Taylor et al., 1988). On the other hand, the syringe filtration and centrifugation techniques need a reasonable time, usually 4.0 - 7.5 minutes, while the vacuum filtration technique requires over 40 minutes for the detection of $S$. haematobium infection. Although haematouria and the macro-technique need no laboratory equipment and require the least time, they have many significant problems. First, as qualitative tools, these methods cannot be used for the assessment of transmission pressure nor for the evaluation of post-interventional activities for bilharzia; second, they are prone to many false-negatives in cases of light infection and false-positives in areas where renal or urinary tract complications are endemic; third for young females, blood associated with the menstrual cycle might act as a confounding factor, thus increasing the number of false-positives. 
The present study confirmed that the syringe filtration, centrifugation and vacuum filtration techniques are very sensitive in the qualitative and quantitative screening of $S$. haematobium infection. The first two methods are easy to perform in the laboratory as well as in the field, in addition, to the time factor advantage mentioned above. Furthermore, all the equipment required for the two techniques can be washed immediately and used repeatedly for screening. Unfortunately, despite the high efficacy of the vacuum filtration technique its usefulness is limited by the time needed for performing the technique. Thus, it is not recommended for use in epidemiological surveys or at hospital laboratories, but might be useful for the confirmation of screening at a limited level.

For fecal analysis, the wet mounting direct smear technique is the simplest and the easiest and requires only 4.5 - 5.0 minutes, per fecal specimen for the detection of $S$. mansoni and other intestinal parasites. Based on the aforementioned characteristics, this technique is performed in all public and private medical laboratories in Sudan. However, in the two study areas, the wet mounting technique proved to be the poorest of the five screening tools assessed for the detection of $S$. mansoni, infection rate and worm burden. The method is capable of detecting moderate and heavy infections, but light infections will be overlooked. This method is recommended for the diagnosis of intestinal schistosomiasis if no other techniques are available. However, the technique is not recommended for the assessment of transmission pressure nor for the evaluation of control projects.

The modified Kato (template \& injection), Kato-Katz and ether-formalin techniques were the most sensitive tools for the detection of S. mansoni, Enterobius and Ascaris infection rates and worm burden. The first two tools need a reasonable time, 7.0 - 10.5 minutes, while the other last two require $10-14$ minutes for the detection of all intestinal parasites. Although the modified Kato technique has been considered as a good method for measuring intensity, it seems that its use in this way relies on the composition of the original fecal sample. In fact, the fiber content of human stool varies seasonally with the availability of different foods, vegetables and fruits. These include different salads, mango, orange, grapefruit, guava, sugar cane and others.

The Kato-Katz Technique (cellophane-thick smear technique) for stool examination, which was introduced in 1954 by Kato and Miura, has proved very satisfactory in Japan, and has also been modified and improved by many researchers (Martin and Beaver, 1968; Borda and Pellegrine, 1971; Katz et al., 1972). In this study, the technique proved to be an efficient means of diagnosing intestinal schistosomiasis and other intestinal helminthes. This technique can be employed in the field, the samples stored in microscopic slide boxes and shipped over long distances, for examination in a central laboratory if required. During epidemiological work in the Sudan, in view of the difficulty of obtaining cellophane, it can be replaced by a thick glass coverslip, and a syringe can be used as a mean of calibrating the stool sample (Teesdale and Amin, 1976). Ahmed, (2006), modified the technique by using a template (40 milligrams) instead of a syringe (25 milligrams), thus increasing the efficacy of the technique without needing to use malachite green. The efficacy of the ether-formalin technique was higher than that of the direct smear technique, but both the two modified Kato techniques and the Kato Katz methods were found to be less effective in screening for intestinal helminths. The two modified Kato techniques failed to detect the intestinal protozoa, G. lamblia and E. histolytica, and the etherformalin technique proved to be the most sensitive technique in their detection. In conclusion, 
the direct smear technique for diagnosis of stool samples proved to under-represent the proportion of parasitic infections. For this reason, the use of the modified Kato techniques is highly recommended, in both public and private medical laboratories.

\section{ACKNOWLEDGEMENTS}

The authors would like to thank the families and children of El-Kalakla and El-Rimeila villages for their help and cooperation in conducting this study. Thanks are also due to the staff of the Schistosomiasis Research Laboratory, University of Khartoum, for their kind assistance.

\section{REFERENCES}

[1] Ahmed, A. 2006. Schistosomiasis in sugar cane schemes, Sudan. Sudan J Nat Sci, (4B): 1-11.

[2] Ansong, D., Alder, S.C., Crookston, B.T., Beck, C., Gyampomah, T. et al. 2011. Role of diagnostic testing in schistosomiasis control programs in rural Ghana. J Bacteriol Parasitol, 2: 115.

[3] Borda, C.E., Pellegrino, J. 1971. An improved thick smear technique for diagnosis of Schistosoma mansoni infection, Revisto institute. Med Trop, Sao Paulo, 13: 71-75.

[4] Colley, D.G., Bustinduy, A.L., Secor, W.E., King, C.H. 2014. Human schistosomiasis. Lancet, 383(9936):2253-2264.

[5] Corstjens, P.L., Nyakundi, R.K., de Dood, C.J., Kariuki, T.M., Ochola, E.A., Karanja, D.M., Mwinzi, P.N., van Dam, G.J. 2015. Improved sensitivity of the urine CAA lateralflow assay for diagnosing active Schistosoma infections by using larger sample volumes. Parasit Vectors, 8, doi:10.1186/s13071-015-0857-7.

[6] Dazo, B.C., Biles, J.E. 1974. Two new field techniques for detection and counting of Schistosoma haematobium eggs in urine samples, with an evaluation of both methods. Bull World Health Organ, 51: 399-408.

[7] De Jonge, N., Gryseels, B., Hilberath, G.W., Polderman, A.M., Deelder, A.M. 1988. Detection of circulating anodic antigen by ELISA for seroepidemiology of schistosomiasis mansoni. Trans R Soc Trop Med Hyg, 82(4): 591-594.

[8] Fatiregun, A.A., Osungbade, K.O., Olumide, A.E. 2009. Cost-effectiveness of screening methods for urinary schistosomiasis in a school-based control programme in Ibadan, Nigeria. Health Policy, 89: 72-77.

[9] Kato, K., Miura, M. 1954. Comparative examinations of faecal samples. Jpn J Parasitol, 3: 70-75.

[10] Katz, N., Chaves, A., Pellegrino, J. 1972. A simple device for quantitative stool thicksmear technique in Schistosomiasis mansoni. Rev Inst Med Trop Sao Paulo, 14: 397-400.

[11] Magnussen, P., Ndawi, B., Sheshe, A.K., Byskov, J., Mbwana, K. 2001. The impact of a school health programme on the prevalence and morbidity of urinary schistosomiasis in Mwera Division, Pangani District, Tanzania. Trans R Soc Trop Med Hyg, 95: 58-64.

[12] Martin, L.K., Beaver, P.C. 1969. Evaluation of Kato thick smears technique for quantitative diagnosis of helminth infections. Am J Trop Med Hyg, 17: 382-391.

[13] Sturrock, R.F. 2001. Schistosomiasis epidemiology and control: How did we get here and where should we go? Mem Inst Oswaldo Cruz, 96: 17-27. 
[14] Taylor, P.T., Murare, H.M., Manomano, K. 1988. Efficacy of low doses of praziquantel for Schistosoma mansoni and S. haematobium. J Trop Med Hyg, 91:13-17.

[15] Teesdale, C.H., Amin, M.A. 1976. Comparison of the Bell technique, a modified Kato thick smear and digestion method for the field diagnosis of S. mansoni. J Helminthol, 50: 17-20.

[16] van Dam, G.J., Wichers, J.H., Ferreira, T.M., Ghati, D., van Amerongen, A. 2004 . Diagnosis of schistosomiasis by reagent strip test for detection of circulating cathodic antigen. J Clin Microbiol, 42: 5458-5461.

[17] World Health Organization, 2016. Accelerating progress on HIV, tuberculosis, malaria, hepatitis and neglected tropical diseases. A new agenda for 2016-2030. 